\title{
Methotrexate-loaded biodegradable nanoparticles: preparation, characterization and evaluation of its cytotoxic potential against U-343 MGa human neuronal glioblastoma cells
}

\author{
KRANTI P MUSMADE ${ }^{1}$, PRAFUL B DESHPANDE ${ }^{1}$, PRASHANT B MUSMADE ${ }^{1}$, \\ M NASEER MALIYAKKAL ${ }^{1}$, A RANJITH KUMAR ${ }^{2}$, M SREENIVASA REDDY ${ }^{1}$ and \\ N UDUPA ${ }^{1, *}$ \\ ${ }^{1}$ Manipal College of Pharmaceutical Sciences, Manipal 576 104, India \\ ${ }^{2}$ Department of Pharmaceutical Sciences, South Dakota State University, South Dakota 57007, USA
}

MS received 15 March 2013; revised 27 July 2013

\begin{abstract}
Nanoparticles represent one of the attractive alternatives in the effective treatment of cancer chemotherapy. In the present work, formulation and development of a novel methotrexate (MTX)-loaded biodegradable nanoparticles using poly(D,L-lactide-co-glycolide) (PLGA) was carried out. The prepared nanoparticles were evaluated for physicochemical properties such as particle size, zeta potential, release studies, etc and also evaluated for its in vitro cytotoxic potential against U-343 MGa human neuronal glioblastoma cells. Particle size of optimized formulation was $<200 \mathrm{~nm}$. There was a considerable decrease in cell viability and enhancement in cytotoxic activity of MTX-loaded nanoparticles compared to MTX alone when tested against U-343 MGa human neuronal glioblastoma cells.
\end{abstract}

Keywords. Methotrexate; PLGA 50:50; nanoparticles; cytotoxic activity.

\section{Introduction}

In recent years, there has been a rapid increase in nanotechnology applications to medicine in order to prevent and treat several life-threatening diseases. Delivery of drugs through nanoparticulate-based drug carriers has attained an attractive alternative mainly, in the treatment of different types of malignancies. These sub-micronic particles have potential to target the tumour sites passively, through enhanced permeation retention effect. Nanoparticles also offer the advantage of targetted or sitespecific delivery of chemotherapeutics and other drugs to affected cells over an extended period of time thereby increasing efficacy, while reducing toxic side effects (De Jong and Borm 2008). Advances in nanotechnology assist drugs to be delivered in the ways that preserve their efficacy and to precise therapeutic targets, creating a host of opportunities for cancer chemotherapy (Singh et al 1997; Singh and Udupa 1997).

Methotrexate (MTX), a folic acid antagonist is commonly used in the treatment of neoplastic disorders (Zhang et al 2005). MTX belongs to the BCS-IV class which warrants its absorption in vivo. MTX is a cell cycle phase specific drug, hence prolonged exposure of the drug to the cancer cells is essential for optimum therapeutic effect (Singh et al 1997; Singh and Udupa 1997).

*Author for correspondence (n.udupa@manipal.edu)
MTX shows hematological effects like leucopenia, thrombocytopenia, anemia and haemorrhage from various sites in single-dose intravenous administration and also some hepatic toxicity by administering as conventional dosage forms. Sustained and targetted delivery of MTX will reduce these toxicities considerably, by maintaining a low and constant level of drug in the blood. A nanoparticulate approach could be assumed to reduce its toxic effects as well as to achieve anticipated sustained release properties. In recent years, biodegradable polymeric nanoparticles have attracted considerable attention as potential drug delivery systems (Soppimath et al 2001). The aim of the present study was to formulate biodegradable polymeric nanoparticles of MTX to enhance its delivery efficacy as well as to improve its pharmacokinetic properties.

\section{Materials and methods}

\subsection{Materials}

Methotrexate was obtained as gift sample from Biochem Industries, Mumbai, India. Polymer, poly(D,L-lactide-coglycolide) (PLGA) $(50: 50)$ (Resomer $\left.{ }^{\circledR} 503 \mathrm{H}\right)$ was purchased from Boehringer Ingelheim Pharma Gmbh \& Co, Germany. Poly(vinyl alcohol) (cold water soluble) with 87-90\%, hydrolysed, molecular weight 30,000-70,000, Pluronic F 68, Pluronic F 127 and MTT reagent were 
purchased from Sigma Aldrich, St. Louis, MO, USA and all other reagents used were of analytical grade.

\subsection{Preparation of nanoparticles}

In the present study nanoparticles of MTX were prepared by emulsification solvent evaporation method (Xua et al 2005). In brief, polymer and MTX were dissolved in acetone under high speed homogenization (Polytron Mixer, Kinematica) at $1000 \mathrm{rpm}$. The polymeric solution was probe sonicated for 2 min and slowly added to the aqueous phase containing surfactant solution using high speed homogenization at $7000 \mathrm{rpm}$ for $10 \mathrm{~min}$. Resulting O/W emulsion was again probe sonicated at $40 \mathrm{~W}$ amplitude for $5 \mathrm{~min}$ in ice water bath. The emulsion was kept for stirring on a magnetic stirrer at $1200 \mathrm{rpm}$ for complete evaporation of organic solvent. After complete evaporation of the solvent, the suspension was centrifuged at $20,000 \mathrm{rpm}$ for $30 \mathrm{~min}$. The pellet at the bottom was reconstituted in a solution containing mannitol as a cryoprotectant. The suspension was deep-freezed at $-80{ }^{\circ} \mathrm{C}$ for $8 \mathrm{~h}$ and lyophilized with vacuum pressure of $<50$ mTorr and at a temperature of $-40{ }^{\circ} \mathrm{C}$ for $48 \mathrm{~h}$. Supernatant was analysed for free drug (unentrapped) using in-house developed RP-HPLC method.

\subsection{Characterization of nanoparticles}

The prepared nanoparticles were characterized for particle size (PS), polydispersity (PDI), zeta potential (ZP), entrapment efficiency, DSC, scanning electron microscopy (SEM), percentage yield, in vitro drug release studies and in vitro cytotoxicity assay using U-343 MGa human neuronal glioblastoma cells.

2.3a Particle size, polydispersity index and zeta potential: These parameters were analysed using the Zetasizer Nano ZS (Malvern Instruments Ltd, UK) instrument utilizing dynamic light scattering (DLS) technique. The zeta potential of a particle is the overall charge that the particle acquires in a particular medium. In this technique, a voltage was applied across a pair of electrodes at either end of a cell containing the particle dispersion. Charged particles were attracted to the oppositely charged electrode and their velocity was measured and expressed in unit field strength as their electrophoretic mobility. The prepared suspensions were diluted in Milli$\mathrm{Q}$ water and placed in measurement cell for analysis $(n=3)$ (Averineni et al 2012).

2.3b Determination of drug encapsulation efficiency by HPLC: An analytical method of MTX has been developed for the determination of encapsulation efficiency. High performance liquid chromatography system, HPLC LC (Shimadzu, Kyoto, Japan) equipped with UV-detector was employed for the development of RP-HPLC method.
Encapsulation efficiency $(\%)=$

$\frac{\text { Amount of drug in nanoparticles }(\mathrm{mg})}{\text { Initial amount of drug }(\mathrm{mg})} \times 100$.

2.3c Scanning electron microscopy (SEM): The shape and surface characteristics of the nanoparticles were studied by scanning electron microscopy (JSM-T20, Kyoto, Japan). An appropriate sample of nanoparticles was mounted on metal (aluminium) stubs, using double-sided adhesive carbon tape and fractured with a razor blade (Reddy et al 2004). The samples were sputter-coated with gold/palladium for $120 \mathrm{~s}$ at $14 \mathrm{~mA}$ under argon atmosphere for secondary electron emissive SEM and observed for morphology at an acceleration voltage of $15 \mathrm{kV}$.

2.3d Percentage yield: The percentage yield was determined according to formula given below

$$
\% \text { Yield }=\frac{\text { Weight of nanoparticles }}{\text { Weight of drug }+ \text { Weight of polymer }} \text {. }
$$

2.3e DSC studies: Closed vials containing drug excipient blends were charged in stability chamber at $25^{\circ} \mathrm{C} / 60 \%$ $\mathrm{RH}$ for a period of 1 month and observed for change in physical attributes. Controlled samples were kept at refrigerated conditions $\left(2-8{ }^{\circ} \mathrm{C}\right)$. The observations are recorded and DSC thermograms were provided. These studies were carried out to assess interaction between various polymers and drug and to confirm the presence of drug in the nanoparticles either in its crystalline or amorphous form.

2.3f In vitro drug release studies: The in vitro drug release studies for the optimized formulation was carried out in dialysis tubing using phosphate buffer $\mathrm{pH} 6.8$ at $37 \pm 0.5{ }^{\circ} \mathrm{C}$ and at $50 \mathrm{rpm}$. In vitro drug release was assessed by intermittently sampling at pre-determined time intervals $(0.5,0.75,1,2,3,4,6,8,12,24,48$ and $72 \mathrm{~h}$ ) and was replaced with fresh $\mathrm{pH} 6.8$ phosphate buffer. The withdrawn sample was centrifuged at $5000 \mathrm{rpm}$ for $2 \mathrm{~min}$, supernatant was filtered through $0.45 \mu \mathrm{m}$ membrane filter and injected to HPLC system by using HPLC equipped with UV detector at $307 \mathrm{~nm}$. The amount of MTX released in each sample was determined using a calibration curve; the reported values are averages of three replicates $(n=3)$. Results of in vitro drug release studies obtained were tabulated and shown graphically as cumulative \% drug release vs time.

2.3g In vitro cytotoxicity assay: In vitro cytotoxicity assay was carried out using human neuronal glioblastoma cells (U343) by MTT (3-[4,5-dimethylthiazol-2-yl]-2,5diphenyl tetrazolium bromide) assay. Free MTX and blank nanoparticles unloaded with MTX were also used for relative cytotoxicity studies. Briefly, cells were cultured in healthy conditions in Dulbecco's modified eagle medium (DMEM) with 10\% fetal bovine serum (FBS) as 


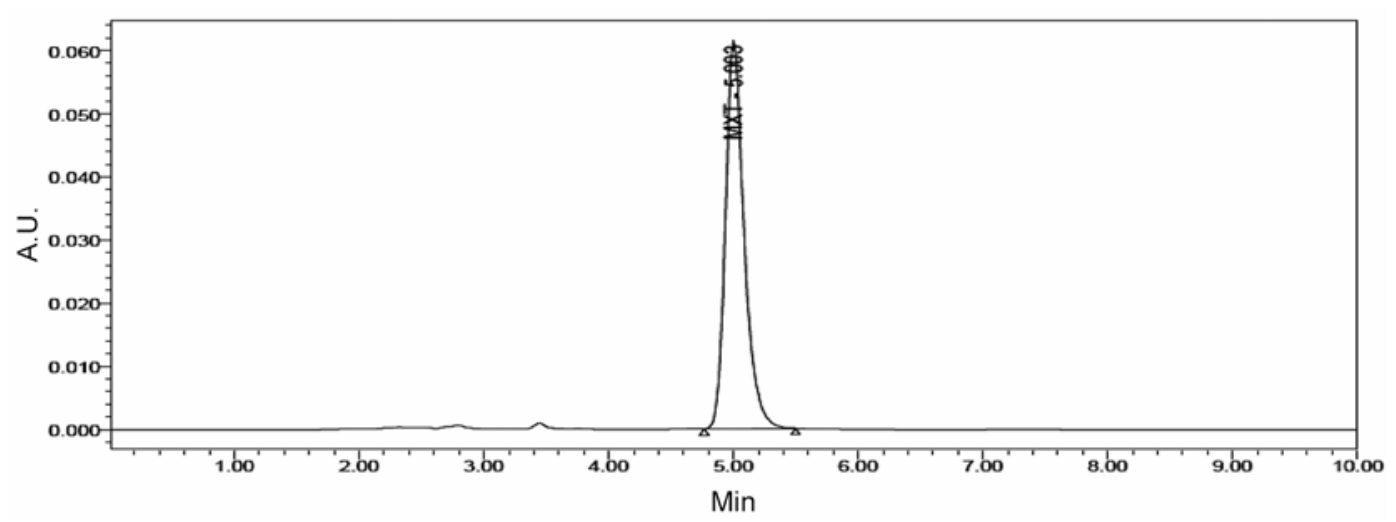

Figure 1. Representative chromatogram of methotrexate by HPLC.

monolayer culture in humidified $37^{\circ} \mathrm{C} \mathrm{CO}_{2}$ incubator. At log phase around $70 \%$ confluency, cells were trypsinized and optimal cell numbers were seeded in a 96 well plate and incubated for $24 \mathrm{~h}$. MTX nanoparticles (F7) were resuspended in sterile IX PBS and serially diluted in DMEM with $10 \%$ FBS to get a final concentration of $0 \cdot 1$, $0 \cdot 5,1,5$ and $50 \mu \mathrm{M}$. After $24 \mathrm{~h}$ of incubation, cells were treated with MTX and MTX nanoparticles at these concentrations for $72 \mathrm{~h}$ and 7 days, respectively. At the end of the treatment, absorbance of formazan in DMSO was measured at $570 \mathrm{~nm}$ using microtiter reader (Pedeboscq et al 2007).

\section{Results and discussion}

\subsection{Analytical method development for estimation of methotrexate}

A rapid sensitive analytical method for the determination of MTX has been developed and validated. High performance liquid chromatography system, HPLC LC (Shimadzu, Kyoto, Japan) equipped with UV-detector was employed for RP-HPLC method development (figure $1)$. The sample was analysed by reverse phase $C_{18}$ Grace Vydac $(250 \times 4.6 \mathrm{~mm}, 5 \mu)$ as stationary phase and acetonitrile: $\mathrm{pH} 6$ buffer solution $(10: 90 \% \mathrm{v} / \mathrm{v})$ as a mobile phase at a flow rate of $1 \mathrm{~mL} / \mathrm{min}$. Quantification was achieved with ultraviolet detection at $307 \mathrm{~nm}$. The retention time of MTX was found to be $5 \pm 0.1 \mathrm{~min}$. The developed method was validated for various parameters as per the ICH guidelines.

\subsection{Formulation development}

From the above method, the nanoparticles thus obtained were dry, free-flowing and readily redispersible when reconstituted with Milli-Q water. The percentage yield of MTX incorporated PLGA nanoparticles were obtained in the range of $56 \cdot 32-90 \cdot 41 \%$. Formulation optimization batches were taken to obtain desired particle size, entrapment efficiency and \% drug release. Drug to polymer ratio and concentration of stabilizer were found to influence the physicochemical properties of the MTX-loaded nanoparticles. The average particle size for optimum batch of nanoparticles was found to be $115-270 \mathrm{~nm}$, PDI below 0.50 and $\mathrm{ZP}$ below $-30 \mathrm{mV}$. The $\%$ encapsulation efficiency varied between 8 and 16\% for all the batches. The low percentage encapsulation efficiency was due to difference in osmotic pressure between the two phases. The osmotic pressure difference increased with increase in MTX loading, leading to a rupture of the lipophilic droplets and an exchange between the internal and external aqueous phases, with a consequent loss of MTX. It is also possible that the polymer does not precipitate quickly enough and loss of MTX occurs. The solvent evaporation time and the nature of continuous phase are the tools to improve encapsulation (Patel 2010). Tables 1 and 2 show physiochemical properties of MTX formulation. Particle size and zeta potential distributions of optimized MTX nanoparticles are given in figure 2.

3.2a Influence of surfactant concentration on various formulation parameters: The particle size increased with increasing surfactant concentration although the increase was not significant $(p>0 \cdot 05)$. Entrapment efficiency decreased with increasing PVA concentration. Based on particle size distribution and entrapment efficiency, the $0.5 \%$ PVA concentration was selected for further studies (figure 3).

3.2b Influence of drug: polymer ratio on various formulation parameters: The particle size and entrapment efficiency were increased with increasing the drug: polymer ratio. The particle size increased with increasing concentration of PLGA dissolved in a fixed volume of solvent although, the increase was not significant $(p>0.05)$. Slight fusion of semi-formed particles might have resulted due to increased frequency of collision, when PLGA concentration was increased (figure 4). 
Table 1. Physiochemical properties of optimized formulation.

\begin{tabular}{lccccc}
\hline $\begin{array}{l}\text { Sl. } \\
\text { no. }\end{array}$ & $\begin{array}{c}\text { Drug : polymer } \\
\text { ratio }\end{array}$ & $\begin{array}{c}\text { Surfactant conc. } \\
(\% \mathrm{w} / \mathrm{v})\end{array}$ & $\begin{array}{c}\text { Particle size } \\
(\mathrm{nm})\end{array}$ & $\begin{array}{c}\text { Zeta potential } \\
(\mathrm{mV})\end{array}$ & $\begin{array}{c}\text { \% entrapment } \\
\text { efficiency }\end{array}$ \\
\hline 1 & $1: 100$ & PVA $(0.5 \% \mathrm{w} / \mathrm{v})$ & 199.7 & $-22 \cdot 2$ & 15.43 \\
\hline
\end{tabular}

Table 2. Formulation of methotrexate biodegradable nanoparticles.

\begin{tabular}{lccccccc}
\hline $\begin{array}{l}\text { Batch } \\
\text { code }\end{array}$ & Polymers & $\begin{array}{c}\text { Drug }: \text { polymer } \\
\text { ratio }\end{array}$ & Surfactant & $\begin{array}{c}\text { Surfactant conc. } \\
(\% \mathrm{w} / \mathrm{v})\end{array}$ & $\begin{array}{c}\text { Particle size } \\
(\mathrm{nm})\end{array}$ & $\begin{array}{c}\text { Zeta potential } \\
(\mathrm{mV})\end{array}$ & $\begin{array}{c}\text { \% Entrapment } \\
\text { efficiency }\end{array}$ \\
\hline F1 & PLGA & $1: 5$ & PVA & $0 \cdot 5$ & $115 \cdot 8$ & $-23 \cdot 3$ & $9 \cdot 201$ \\
F2 & PLGA & $1: 10$ & PVA & $0 \cdot 5$ & $126 \cdot 9$ & $-14 \cdot 7$ & $8 \cdot 269$ \\
F3 & PLGA & $1: 20$ & PVA & $0 \cdot 5$ & $144 \cdot 9$ & $-19 \cdot 7$ & $8 \cdot 810$ \\
F4 & PLGA & $1: 40$ & PVA & $0 \cdot 5$ & $164 \cdot 5$ & $-18 \cdot 8$ & $9 \cdot 906$ \\
F5 & PLGA & $1: 60$ & PVA & $0 \cdot 5$ & $188 \cdot 2$ & $-26 \cdot 3$ & $9 \cdot 032$ \\
F6 & PLGA & $1: 80$ & PVA & $0 \cdot 5$ & $194 \cdot 6$ & $-27 \cdot 4$ & $13 \cdot 25$ \\
F7 & PLGA & $1: 100$ & PVA & $0 \cdot 5$ & $199 \cdot 7$ & $-22 \cdot 2$ & $15 \cdot 43$ \\
F8 & PLGA & $1: 125$ & PVA & $0 \cdot 5$ & $233 \cdot 6$ & $-21 \cdot 9$ & $12 \cdot 80$ \\
F9 & PLGA & $1: 150$ & PVA & $0 \cdot 5$ & $268 \cdot 0$ & $-24 \cdot 7$ & $14 \cdot 60$ \\
F10 & PLGA & $1: 100$ & PVA & $0 \cdot 25$ & $194 \cdot 6$ & $-25 \cdot 9$ & $14 \cdot 85$ \\
F11 & PLGA & $1: 100$ & PVA & $1 \cdot 0$ & $197 \cdot 9$ & $-21 \cdot 1$ & $12 \cdot 44$ \\
F12 & PLGA & $1: 100$ & PVA & $1 \cdot 5$ & $211 \cdot 7$ & $-26 \cdot 5$ & $11 \cdot 86$ \\
F13 & PLGA & $1: 100$ & PVA & $2 \cdot 0$ & $208 \cdot 7$ & $-23 \cdot 9$ & $10 \cdot 55$ \\
F14 & PLGA & $1: 100$ & F 68 & $0 \cdot 5$ & $137 \cdot 9$ & $-33 \cdot 9$ & $8 \cdot 19$ \\
F15 & PLGA & $1: 100$ & F 68 & $1 \cdot 0$ & $126 \cdot 6$ & $-34 \cdot 7$ & $5 \cdot 08$ \\
F16 & PLGA & $1: 100$ & F 68 & $1 \cdot 5$ & $127 \cdot 0$ & $-36 \cdot 2$ & $6 \cdot 90$ \\
F17 & PLGA & $1: 100$ & F 68 & $0 \cdot 25$ & $114 \cdot 7$ & $-40 \cdot 4$ & $8 \cdot 70$ \\
F18 & PLGA & $1: 100$ & F127 & $0 \cdot 25$ & $135 \cdot 7$ & $-24 \cdot 6$ & $7 \cdot 88$ \\
F19 & PLGA & $1: 100$ & F127 & $0 \cdot 5$ & $157 \cdot 3$ & $-30 \cdot 9$ & $7 \cdot 14$ \\
F20 & PLGA & $1: 100$ & F 127 & $1 \cdot 0$ & $171 \cdot 8$ & $-28 \cdot 4$ & $7 \cdot 69$ \\
\hline
\end{tabular}

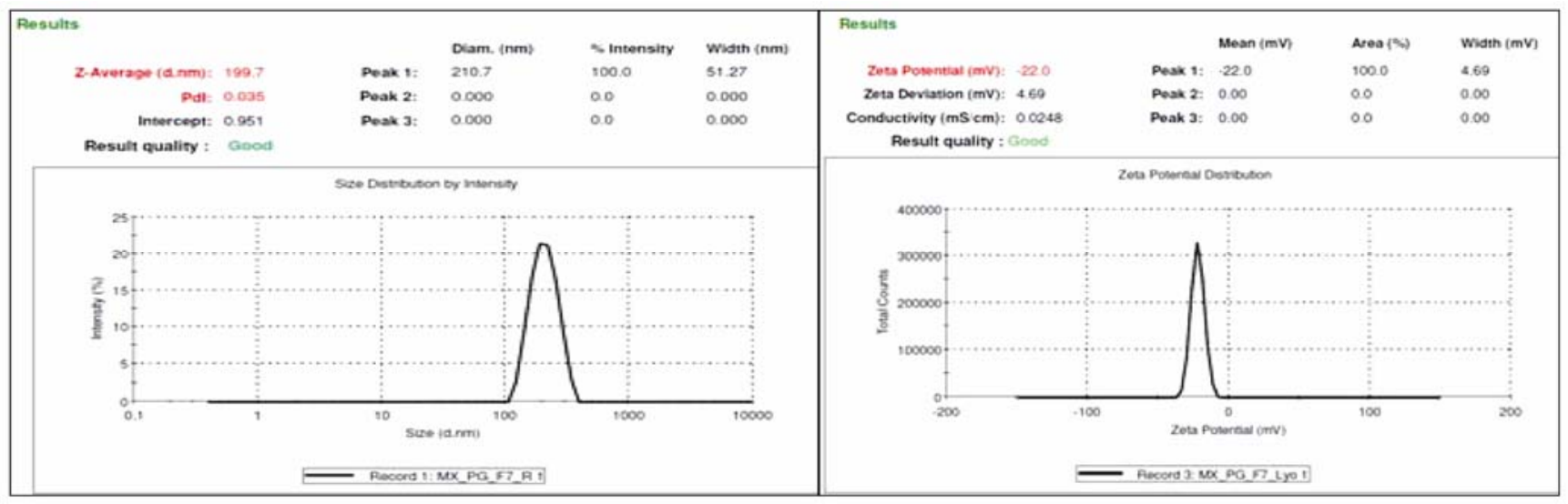

Figure 2. Particle size analysis and zeta potential of optimized methotrexate nanoparticles.

\subsection{Surface morphology: scanning electron microscopy (SEM)}

SEM revealed that the MTX nanoparticles were smooth and spherical without any aggregation (figure 5).

\subsection{DSC studies}

The thermal peak of the drug and excipients interaction in the formulations was monitored by DSC studies.
DSC thermal curve of samples was compared with pure MTX. It was observed that DSC thermal peak of drug excipient blend is same as that of the pure MTX. The endothermic peak corresponding to the melting point of MTX slightly shifted to $\pm 1^{\circ} \mathrm{C}$. The thermogram of the samples did not show any significant shift in the endothermic peak. DSC thermograms demonstrate absence of characteristic peak of MTX in formulations confirming amorphization of MTX in nanoparticles (figure 6). 

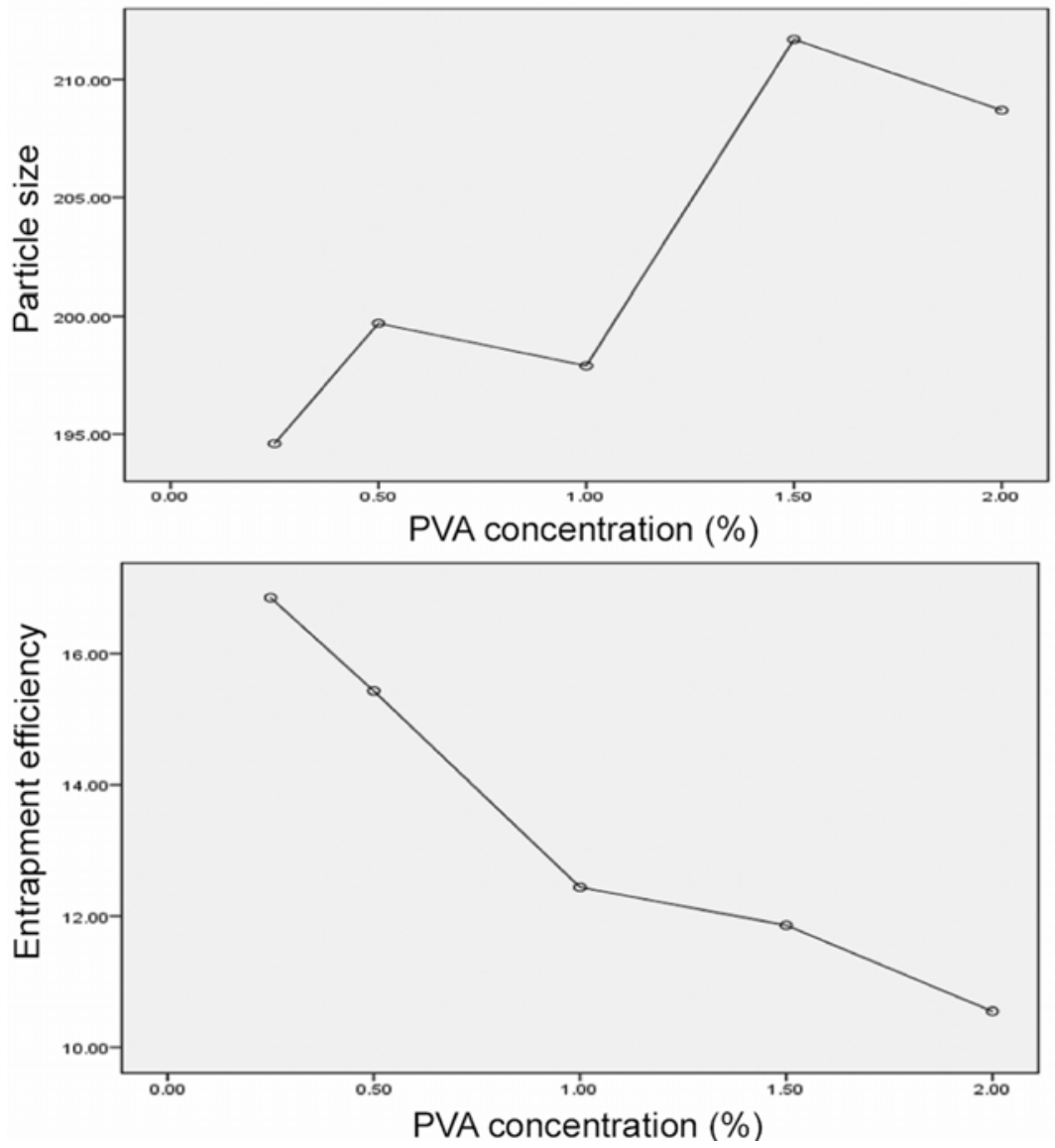

Figure 3. Influence of surfactant concentration on particle size and entrapment efficiency.
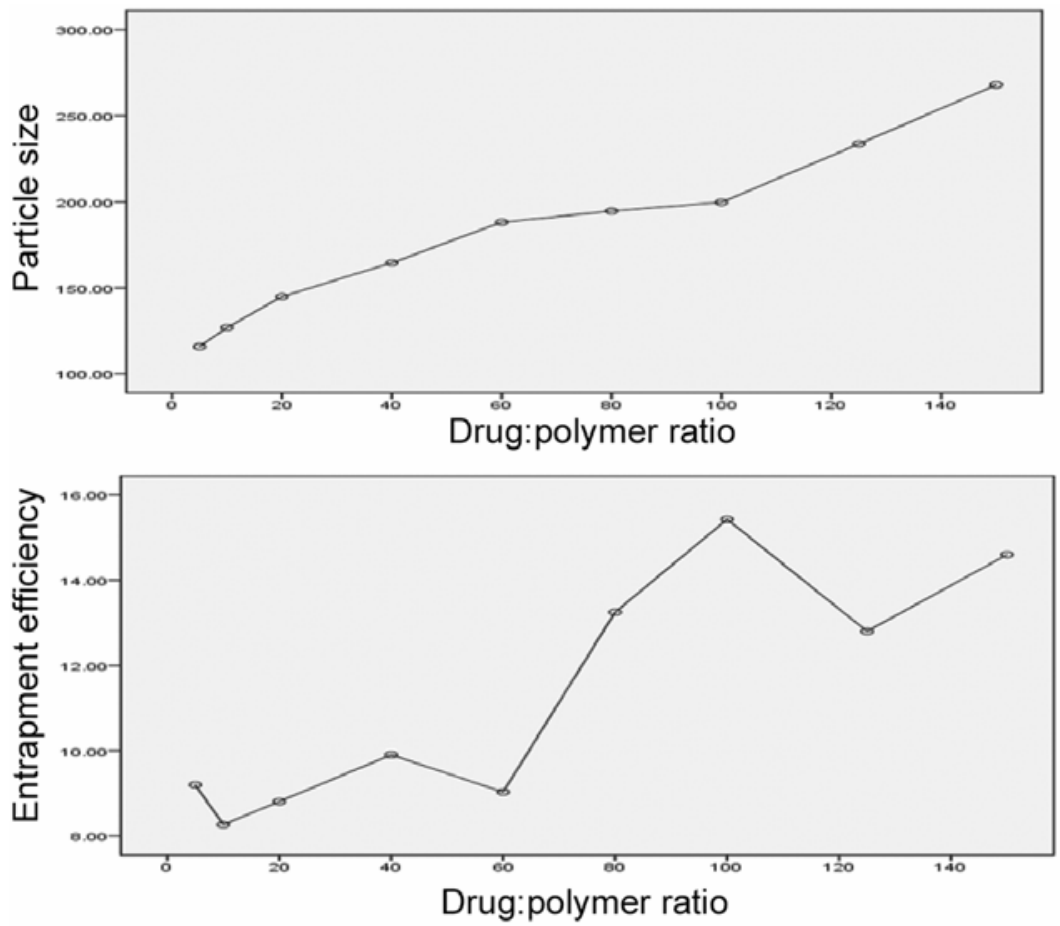

Figure 4. Influence of drug to polymer ratio on particle size and entrapment efficiency. 


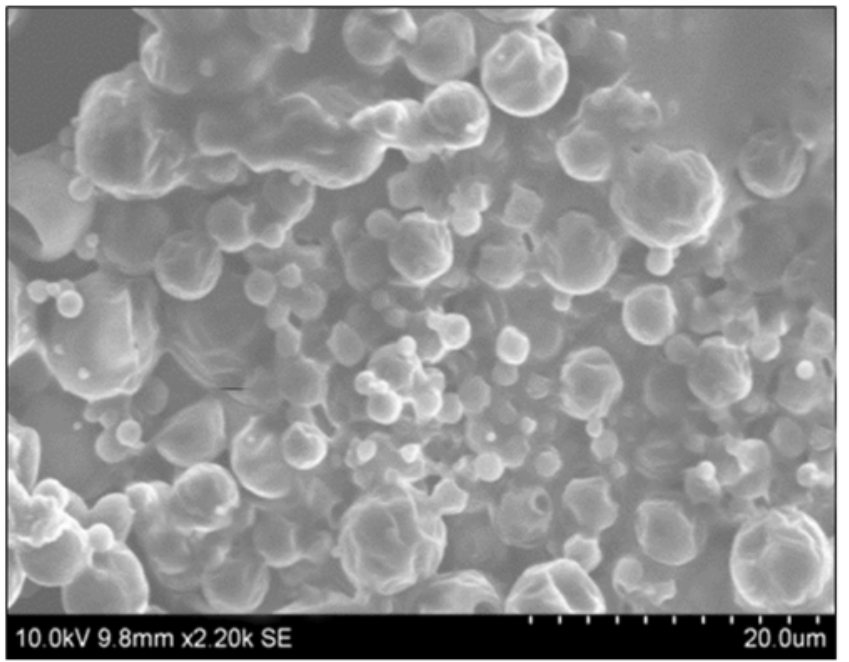

Figure 5. SEM image of methotrexate loaded PLGA nanoparticles.

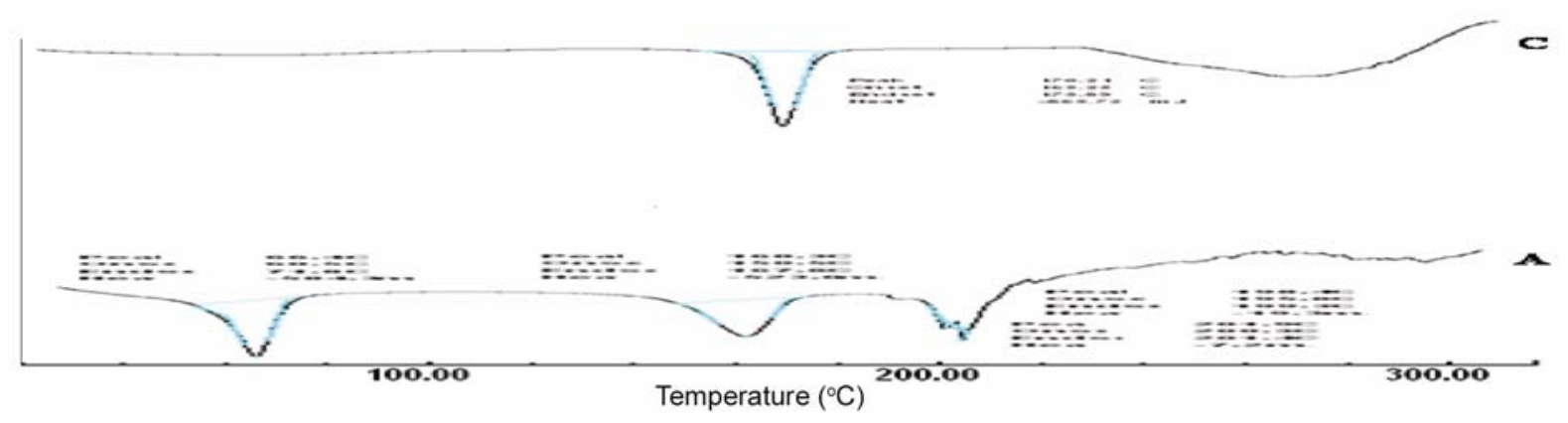

Figure 6. Differential scanning colorimetry of physical mixture and formulation.

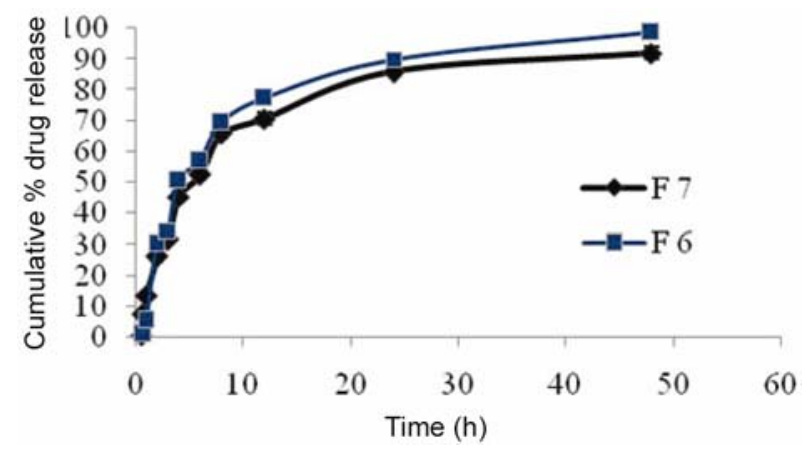

Figure 7. Cumulative percentage drug release of methotrexate nanoparticle.

\subsection{In vitro drug release studies}

Results indicate that the formulation showed initial burst release followed by sustained release of the drug for a prolonged period of time (figure 7). The rapid initial release may be attributed to the fraction of MTX on the surface of nanoparticles. The in vitro drug release results revealed that the prepared PLGA nanoparticles would be able to control drug release for extended period of time.

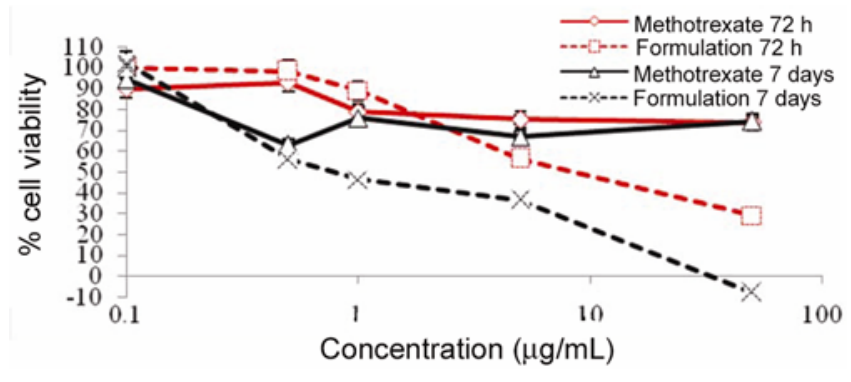

Figure 8. In vitro cytotoxicity studies of methotrexate nanoparticle in human brain cancer cells.

\subsection{Anticancer studies in human brain cancer cells: MTT-based cytotoxicity assay}

Percentage cell viability ( $y$-axis) was plotted against concentration of MTX nanoparticle ( $x$-axis) (figure 8). An $\mathrm{IC}_{50}$ value (concentration of nanoparticles that reduces the viability of cell to 50\%) was determined for MTX and MTX nanoparticles for both $72 \mathrm{~h}$ and 7 days treatment. Results indicate a dose-dependent decline in the percentage cell viability after treatment with MTX or MTX nanoparticles. The result suggests that the polymeric 
nanoparticle releases the drug slowly and have increased retention in the cells as compared to MTX alone. An $\mathrm{IC}_{50}$ value was found to be less for MTX nanoparticles tested as compared to pure drug alone. The cell death was significantly increased with MTX-loaded nanoparticles compared to free MTX, indicating enhanced antitumor activity of nanoparticles against human neuronal glioblastoma cells $(p<0.05)$.

\section{Conclusions}

Our primary results demonstrate that the developed methotrexate-loaded PLGA nanoparticles releasing the drug for prolonged period of time. The developed formulation has better anticancer activity compared to the pure drug.

From this, it is possible to demonstrate nanoparticles as a better vector for the improvement of delivery efficacy of methotrexate.

\section{Acknowledgement}

The authors would like to acknowledge research grant from the Department of Biotechnology, Ministry of Science and Technology, Government of India (Project no. BT/PR10830/NNT/28/114/2008), for providing financial support.

\section{References}

Averineni R K, Shavi G V, Gurram A K, Deshpande P B, Arumugam K, Maliyakkal N, Reddy M S and Udupa N 2012 Bull. Mater. Sci. 35319

De Jong W H and Borm P J A 2008 Int. J. Nanomedicine 3 133

Patel H V, Patel B G and Patel V A 2010 Int. J. Pharm. Res. 2 75

Pedeboscq S, L’Azou B, Liguoro D, Pometan J P and Cambar J 2007 Exp. Toxicol. Pathol. 58247

Reddy H L, Sharma K R, Chuttani K, Mishra A K and Murthy R R 2004 The AAPS J. (Article 23)

Singh U V, Pandey S, Umadevi P and Udupa N 1997 Drug Del. 4101

Singh U V and Udupa N 1997 Acta Helvetiae 72165

Soppimath K S, Aminabhavi T M, Kulkarni A R and Rudzinski W E 2001 J. Control Rel. 701

Xua Z, Gu W, Huang J, Sui H, Zhou Z, Yang Y, Yan Z and Li Y 2005 Int. J. Pharm. 288361

Zhang Y, Jin T and Zhuo R 2005 Coll. Surf. B Biointerf. 44104 